\title{
BMJ Open Longitudinal Study of music Therapy's Effectiveness for Premature infants and their caregivers (LongSTEP): protocol for an international randomised trial
}

To cite: Ghetti C,

Bieleninik $Ł$, Hysing M, et al. Longitudinal Study of music Therapy's Effectiveness for Premature infants and their caregivers (LongSTEP): protocol for an international randomised trial. BMJ Open 2019;9:e025062. doi:10.1136/ bmjopen-2018-025062

- Prepublication history and additional material for this paper are available online. To view these files, please visit the journal online (http://dx.doi. org/10.1136/bmjopen-2018025062).

Received 28 June 2018 Revised 06 March 2019 Accepted 08 July 2019

Check for updates

(C) Author(s) (or their employer(s)) 2019. Re-use permitted under CC BY-NC. No commercial re-use. See rights and permissions. Published by BMJ.

For numbered affiliations see end of article.

Correspondence to

Dr Claire Ghetti;

Claire.Ghetti@uib.no

\section{ABSTRACT}

Introduction Preterm birth has major medical, psychological and socioeconomic consequences worldwide. Music therapy (MT) has positive effects on physiological measures of preterm infants and maternal anxiety, but rigorous studies including long-term follow-up are missing. Drawing on caregivers' inherent resources, this study emphasises caregiver involvement in MT to promote attuned, developmentally appropriate musical interactions that may be of mutual benefit to infant and parent. This study will determine whether MT, as delivered by a qualified music therapist during neonatal intensive care unit (NICU) hospitalisation and/or in home/municipal settings following discharge, is superior to standard care in improving bonding between primary caregivers and preterm infants, parent well-being and infant development. Methods and analysis Design: international multicentre, assessor-blind, $2 \times 2$ factorial, pragmatic randomised controlled trial; informed by a completed feasibility study. Participants: 250 preterm infants and their parents. Intervention: MT focusing on parental singing specifically tailored to infant responses, will be delivered during NICU and/or during a postdischarge 6-month period. Primary outcome: changes in mother-infant bonding at 6-month corrected age (CA), as measured by the Postpartum Bonding Questionnaire. Secondary outcomes: motherinfant bonding at discharge and at 12-month CA; child development over 24 months; and parental depression, anxiety and stress, and infant rehospitalisation, all over 12 months.

Ethics and dissemination The Regional Committees for Medical and Health Research Ethics approved the study (2018/994/REK Nord, 03 July 2018). Service users were involved in development of the study and will be involved in implementation and dissemination. Dissemination of findings will apply to local, national and international levels.

Trial registration number NCT03564184

\section{BACKGROUND}

The global average rate of preterm birth is around $11 \%$ worldwide corresponding to 15 million preterm infants each year. ${ }^{12}$ Over one million die as a direct result of prematurity,
Strengths and limitations of this study

- Following several studies suggesting short-term effects of music therapy (MT) for premature infants, this study will examine longer-term effects up to 2 years.

- Parents will be actively included in MT, with the aim of improving outcomes of both infants and parents.

- MT will be continued after discharge from hospital thus providing a bridge between specialised and primary healthcare.

- As a multinational trial, results will be relevant across a range of settings in diverse societies that enable social support from families.

- Due to the nature of the intervention, participants cannot be blinded. Similarly, most outcomes cannot be blinded because they rely on parent reports.

and another million show lifetime impairments. ${ }^{3}$ With improved survival rates, there is growing concern for long-lasting complications including mental health problems, cognitive impairments and poorer quality of life. ${ }^{14-6}$ Moreover, the economic burden of preterm birth includes increased cost of neonatal care, long-term costs associated with complex health status, and losses in economic productivity over the lifespan. ${ }^{7}$

Premature labour is perceived as a stressful ${ }^{8}$ and traumatic ${ }^{9} 10$ event that can interrupt mothers' antenatal bonding. ${ }^{11}$ Families of premature infants can experience higher levels of stress, anxiety and fear for the infant's safety, as well as insecurity and powerlessness. ${ }^{10} 12$ Mothers are at risk for postpartum depression, post-traumatic stress disorder, feelings of guilt, mourning and lack of selfworth. ${ }^{13}$ Fathers experience significant stress, which may extend beyond the acute phase following the infant's birth. ${ }^{14}$ Supportive and educational interventions provided to 
fathers in the acute phase of the infant's hospitalisation can empower their fathering ability and reduce paternal stress. ${ }^{15}$

Separation due to neonatal intensive care unit (NICU) stay and prolonged hospitalisation may complicate the development of healthy parent-infant bonding and in some cases adversely impact the formation of secure attachment. ${ }^{1016}$ Mother-infant relationship plays a central role in the child's socioemotional development and formation of future intimate relationships. ${ }^{17}{ }^{18}$ Mothers' psychological well-being ${ }^{19}$ or postpartum depression ${ }^{20}$ are important factors affecting parent-infant relationship in the postnatal period. However, even with sensitive parenting, children born very preterm or very low birth weight are at higher risk of demonstrating disorganised attachment than those born full-term, and neurological impairment predicts this disorganised attachment. ${ }^{21}$ Involving fathers in infant care, including skin-to-skin care, during the first days of the infant's life can positively impact the bonding process while improving support to partners, ${ }^{22}$ and can lead to improved self-esteem, feelings of closeness to the infant and perception of more equal parenthood..$^{23}$ Decreasing maternal stress and reducing early separation during NICU hospitalisation may be beneficial for long-term outcomes. ${ }^{24}$

Music therapy (MT) is part of NICU standard treatment in some hospitals in several countries, but supply is usually limited even where that is the case. MT in infant critical care involves the informed use of music and a therapeutic relationship to promote infant development and facilitate bonding with primary caregivers. ${ }^{25}$ MT promotes infant sensory regulation and may contribute to neurological development. ${ }^{26}{ }^{27}$ Incorporating parents as active partners in implementing MT during the NICU stay leads to decreased infant and parent distress, ${ }^{28}$ and may thus promote bonding. ${ }^{29}$ MT beneficially impacts infant physiological parameters, behaviour state, weight gain and feeding ability, and may reduce hospital stay. ${ }^{26}{ }^{30-34}$ There are also some indications of a positive impact of music on sleep for premature infants. ${ }^{31} 3536$ MT provides a culturally sensitive form of family centred care that empowers both mothers and fathers in the care of their premature infants. ${ }^{29}$ MT-based consult-to-parent models, such as the Time Together adaptation of contingent singing into a parent education programme, can help extend benefits to families beyond those most prioritised to receive MT services. ${ }^{37}$

In a rigorous systematic review and meta-analysis of randomised controlled trials (RCTs), a large and favourable effect of MT on respiratory rate and maternal anxiety was confirmed ${ }^{38}$; however, several areas require further investigation. Rigorously designed and adequately powered studies using standardised outcome measures and interventions implemented by music therapists with specialised NICU training are required. Parallel RCTs to evaluate long-term effects of MT, and extending intervention periods past discharge from NICU, are required to assess long-term impact, which is a substantial gap in present knowledge. ${ }^{38}$ Studies examining longitudinal provision of MT tailored to different stages of infant development ${ }^{28}$ and long-term effects of MT on the development of healthy parent-infant bonding and secure attachment are needed..$^{26} 38$

Building on promising results from phase II studies and meta-analyses, Longitudinal Study of music Therapy's Effectiveness for Premature infants and their caregivers (LongSTEP) is the first phase III trial worldwide of MT's effectiveness for premature infants and their caregivers. This trial will determine whether MT as delivered by a qualified music therapist during NICU hospitalisation and/or after discharge to home is superior to standard care in improving bonding between primary caregivers and preterm infants. This project promotes continuity of care by bridging the transition from hospital to home and uses family centred developmental care. Conducting the study in several countries with high levels of parental presence in the NICU, provides sufficient sample size to analyse treatment effects. The results will inform the effective use of MT across levels of healthcare for the participating NICUs in Europe, the Middle East and South America. This study will contribute to an improved understanding of the relation between music and health and will enable evidence-based recommendations for the application of MT in primary healthcare services.

\section{Objectives}

The primary objective of this study is to evaluate the effect of MT on parent-infant bonding at 6-month CA.

Secondary objectives are as follows:

1. To examine effects of MT on premature infants' general and socioemotional development.

2. To examine effects of MT on parents' symptoms of depression, anxiety and stress.

3. To examine medical and social factors of premature infants (sex, gestational age at birth, hearing status) and their parents (bonding, socioeconomic status, breastfeeding, skin-to-skin care) that may be associated with effects of MT.

4. To prepare for examining cost-effectiveness and longer-term evaluation of effects.

We hypothesise that MT plus standard care during or after NICU, compared with standard care alone, will lead to better parent-infant bonding, which in turn may beneficially impact infant development and parent mental health outcomes. Effects of MT may depend on medical and social factors. Clinical effectiveness may be associated with cost-effectiveness and may be sustained in the longer term.

\section{METHODS}

Design

LongSTEP is designed as a $2 \times 2$ factorial, international, multicentre, assessor-blind pragmatic RCT to evaluate the long-term effect of MT in NICU and/or after discharge on premature infants and their primary caregivers across 


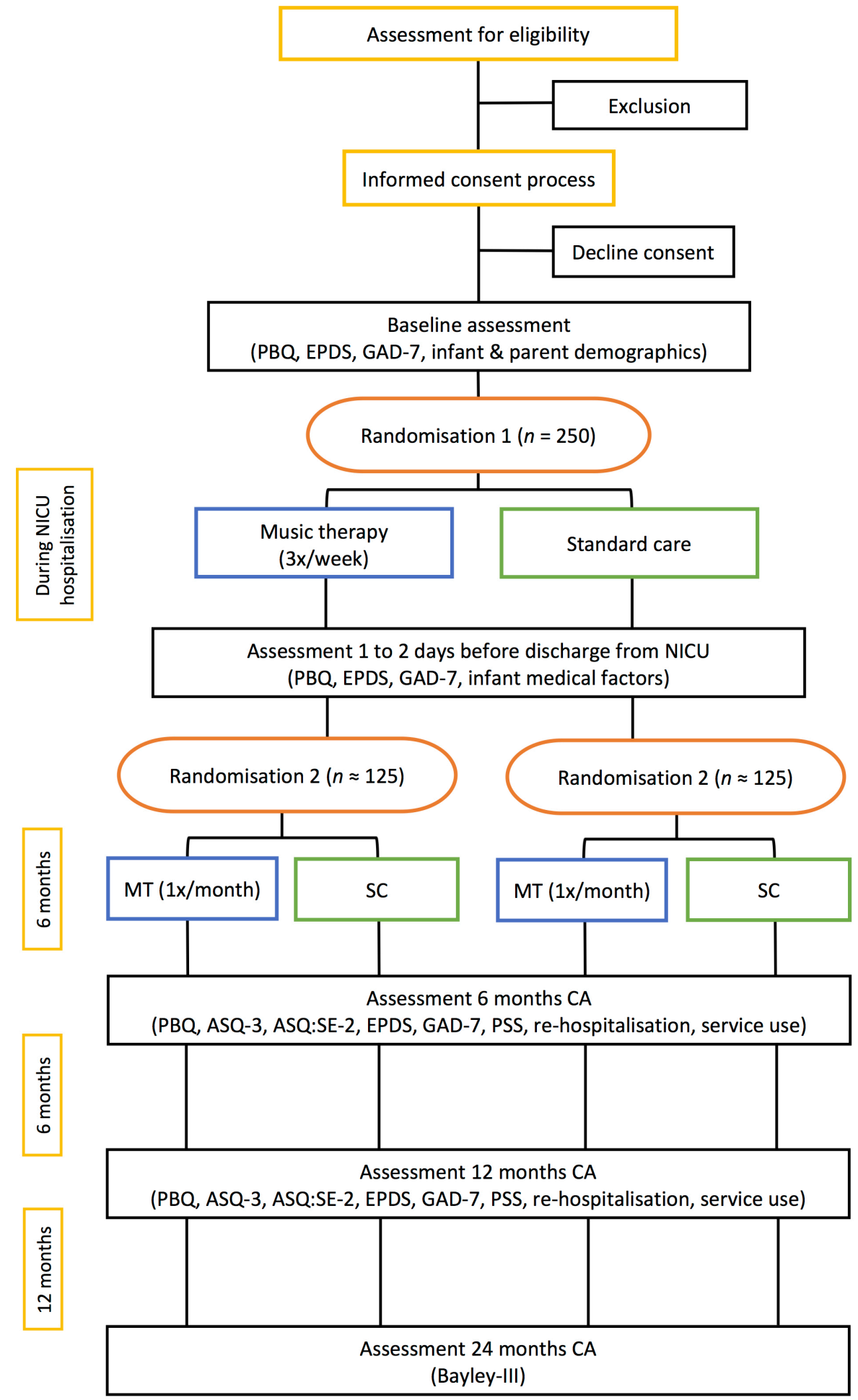

Figure 1 Flow of participants through the study: illustration of the study design. Note: ASQ-3, Ages and Stages Questionnaire (third edition); ASQ:SE-2, Ages and Stages Questionnaire: Social-Emotional (second edition); Bayley-III, Bayley Scales of Infant and Toddler Development (third edition); CA, corrected age (chronological age reduced by number of weeks born preterm); EPDS, Edinburgh Postnatal Depression Scale; GAD-7, Generalised Anxiety Disorder Assessment; PBQ, Postpartum Bonding Questionnaire; PSS, Parental Stress Scale.

a 12-month time period (figure 1). The factorial design entails a two-phase protocol covering both the stay in the NICU and follow-up care after discharge from hospital, with two randomisation time points, and tests the independent effect of each combination: NICU-MT, postdischarge MT, both, or neither, as well as interactions between them.

\section{Patient and public involvement}

Parents of premature infants, hereafter referred to as 'users', play an important role in various aspects of this study. We consulted with users during original development of the project proposal to assure that our research question is highly pertinent to parents of premature 
infants, to estimate level of burden of the intervention and to assess if the intervention is likely to result in benefits that would be of value to users. We incorporated feedback from users in protocol development, both before and after securing funding. We developed a User Advisory Group (UAG) to advise us on selection of outcome measures, feasibility of study design and intervention implementation, and this group will continue to serve an important role during the implementation of the study as described in the section on advisory committees. We will disseminate results of the study to users via popular publications, presentations to parent organisations and informational pamphlets with recommendations for evidence-based practices.

\section{Pilot testing and user feedback}

A feasibility study was conducted at one Norwegian NICU (initiated January 2018) to determine acceptability and suitability of the intervention and study procedures and to obtain parental perspectives in order to inform design of the main study. Pilot testing evaluated feasibility in the areas of participant recruitment, delivery of study conditions, planned assessments and retention, as well as tracking of treatment occurring as part of standard care. Following ethics approval, three families at a Norwegian NICU were offered MT two times per week during NICU hospitalisation and two times per month during the first 3 months after discharge home. Follow-up interviews with parents revealed that parents were willing to take part in MT in the NICU and sing for their infants, and that MT offered a break from typical hospital routines and a different way of 'reading' and relating to their infants. Parents found the self-report outcome measures acceptable and the nature of participation in MT as well as the frequency of sessions feasible and comfortable. Parents stated they would also have been willing to participate in the study if designed as an RCT despite the chance of being allocated to standard care.

A primary aim of the feasibility study was to determine whether the Postpartum Bonding Questionnaire (PBQ) should be retained as a primary outcome in the subsequent main trial. The $\mathrm{PBQ}$ is validated and widely used in the literature, ${ }^{39}$ but we wished to explore its acceptability and perceived relevance with mothers in the feasibility study. While the full results will be published separately, preliminary analysis from the Norwegian feasibility study revealed that the $\mathrm{PBQ}$ scored on relevant items, changed over time, and mothers expressed no particular objections to completing its items. Results from the feasibility study and discussion with parents in our UAG informed our decision to retain the $\mathrm{PBQ}$ as a primary outcome measure for the main study.

The feasibility study also enabled us to test logistical parameters related to postdischarge MT. We provided postdischarge sessions in the home setting, following advice from our UAG. Based on the results from feasibility testing, we decided to adopt the following frequency in the main study to make consistency of sessions more feasible: two times during the first month following discharge from the NICU, and one time per month thereafter for a total period of 6 months.

\section{Trial procedures}

After informed consent and baseline assessment, participants will be randomly assigned to receive either MT plus standard care or standard care alone during their stay at the NICU. The intervention period will start immediately following randomisation and will last until the infant's initial discharge from hospital. Participants will be asked to complete the discharge assessments 1-2 days before discharge to home; for those who are unable to complete them before discharge, assessments will be valid if completed up to 2 weeks after discharge. On completion of discharge assessments, participants will be randomly allocated to receive either postdischarge MT in home or municipal settings along with standard primary care, or standard primary care alone across a 6 -month time period. Standard care may include other early intervention methods of care with the exception of other MT approaches. Participants are free to attend any type of treatment or therapeutic interventions in a postpartum setting, but they will be asked not to attend MT outside the study context through 12-month corrected age (CA; defined as chronological age reduced by number of weeks born before 40 weeks of gestation ${ }^{40}$ ). This time frame is sufficient because the hypotheses of the study concern the effects of early MT during NICU or postdischarge; however, the receipt of any MT during the first 24-month CA outside the study will be recorded. Outcomes will be assessed at several time points: at baseline; 1-2 days before discharge to home (end of MT during NICU); at 6-month CA (end of postdischarge MT; primary endpoint); 12-month and 24-month CA (figures 1 and 2). Treatment fidelity (adherence) will be evaluated by independent raters using audio and video recordings as described further below. The study has been designed in accordance with the SPIRIT 2013 statement (see online supplementary additional file 1). Ethical approval for the study has been granted by The Regional Committees for Medical and Health Research Ethics (2018/994/REK Nord) and supplemental ethical approval from each participating country was secured from relevant ethics committees in those countries prior to commencement of recruitment.

\section{Settings}

To achieve the target sample size, it is necessary to recruit participants from multiple NICUs. Eligible NICUs are located in countries where the culture reflects what could be considered a 'social support' society, where parents are consistently present in the NICU. Eligible NICUs also need to have staff possessing the necessary scientific and clinical expertise to conduct the trial. Current partner sites are located across five countries in Europe, the Middle East and Latin America (Argentina, Colombia, Israel, Norway, Poland), and include eight NICUs (see trial registration data at ClinicalTrials.gov for an updated list). The number of beds in each NICU ranges from 13 to 60 . 


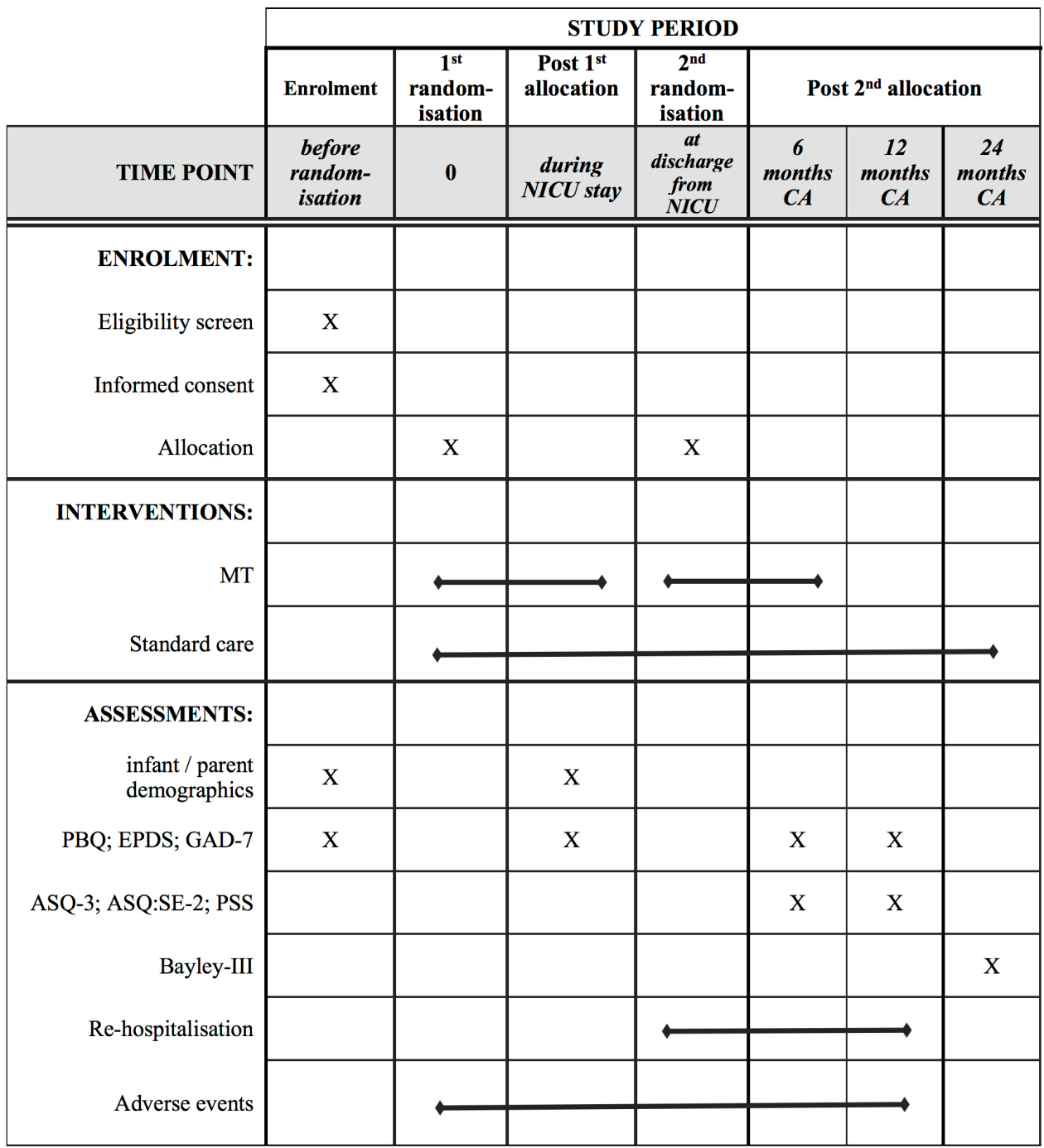

Figure 2 Schedule of enrolment, interventions and assessments. ASQ-3, Ages and Stages Questionnaire (third edition); ASQ:SE-2, Ages and Stages Questionnaire: Social-Emotional (second edition); Bayley-III, Bayley Scales of Infant and Toddler Development (third edition); CA, corrected age (chronological age reduced by number of weeks born preterm); EPDS, Edinburgh Postnatal Depression Scale; GAD-7, Generalised Anxiety Disorder Assessment; PBQ, Postpartum Bonding Questionnaire; PSS, Parental Stress Scale.

\section{Eligibility criteria}

Participants deemed eligible for the trial will meet the following criteria: Infants: born $<35$ weeks' gestational age (GA), of both genders, any ethnicity, from single or multiple pregnancies, and whom the NICU clinical staff judges to be sufficiently medically stable to start MT, and to likely require longer than 2 weeks of hospitalisation from the time of recruitment. In multiple pregnancies, only the first-born infant who achieves medical stability will be included and randomised, while remaining siblings can receive the same interventions for ethical and practical reasons. Infant hearing screenings typically take place prior to discharge from the NICU, thus hearing status may not be known at time of study recruitment. Results of infant hearing screening at discharge will be tracked when available, to facilitate follow-up analysis, but infants who test positive for hearing impairments will still be included in data analysis due to the potential for infant and parent benefit despite such sensory impairment. Primary caregivers: one of the parents/caregivers must provide written, site-specific informed consent before any study procedures occur. Primary caregivers should agree to engage in at least 2 of the 3 MT sessions per week during NICU, and/or in 5 of the 7 MT postdischarge sessions. Primary caregivers should live within reasonable commuting distance from the treating NICU and should have sufficient understanding of the respective national language(s) to answer the questionnaires and participate in MT. Parents who are unable to complete the intervention and/or questionnaires, for example, due to a mental illness, cognitive impairment and so on, will be excluded.

\section{Interventions}

The MT approach in this study is informed by models previously described and tested, including Creative Music Therapy with premature infants and their 
parents, ${ }^{26} 41 \quad 42$ First Sounds: Rhythm, Breath and Lullaby, ${ }^{31} 434$ and other developmentally appropriate and family centred approaches to MT with premature infants and their families. ${ }^{27} 4546$ Like other MT models, ${ }^{414}$ it is based on the notion that infants have a capacity to communicate actively. ${ }^{47}$ As in some newer models, parents are highly involved and the therapeutic benefit is directed towards infant and parents alike. ${ }^{3141446}$ The approach here thus builds on an emerging consensus, but is more explicit in its adaptation to developmental levels and emphasis on infant-parent mutual regulation. ${ }^{49}$ Furthermore, unlike previous studies, ${ }^{38} \mathrm{MT}$ is extended beyond the tertiary care setting to help with the transition to daily life and municipal care. MT focuses on supporting positive relationship formation within the infant-parent dyad by promoting beneficial mutual regulation, and includes psychotherapeutic support of the parents to promote their stabilisation, self-regulation, bonding and restorative experience in alignment with trauma-preventive models. ${ }^{445}$ Key elements of the MT intervention appear in table 1.

\section{MT during NICU}

Participants will be offered individual MT sessions 3 times/week for $\sim 30-40 \mathrm{~min} / \mathrm{session}$, with a maximum of 27 sessions per infant (assuming a maximum NICU stay of 9 weeks). Time spent in active music making with the infant may be shorter than $30 \mathrm{~min}$, depending on infant readiness, and the remaining portion of the session will be devoted to providing psychotherapeutic support for parents and coaching them in how to use their voices therapeutically with their infants. MT will be provided for eligible infants once they achieve sufficient medical stability to begin MT, usually after 26 weeks' postmenstrual age (PMA; defined as GA at birth plus the time since birth $\left.{ }^{40}\right)$. Use of live music will follow recommendations from the American Academy of Pediatrics Committee on Environmental Health ${ }^{51}$ and the Consensus Committee on Recommended Design Standards for Advanced Neonatal Care ${ }^{52}$ namely that noise levels within the NICU should remain $<45 \mathrm{~dB}$ (A weighted), with hourly maximum sound levels (Lmax) $<65 \mathrm{dBA}$, and no $>10 \%$ of each hour (L10) $>50 \mathrm{dBA}$. Prior to the first MT session with the infant, the music therapist will meet with the parents to discuss MT and assess aspects that will inform the course of MT including music preferences and history, cultural values related to music, level of comfort with singing, infant signals and patterns, family strengths and needs, and any challenges and resources that the parents deem relevant. The music therapist will then explain basic elements of the procedures listed below, engage parents in musical demonstration and form a loose plan for how the parents and music therapist will begin MT with the infant. The music therapist will observe the infant during a period of handling (such as during diaper change or transition to parent's arms) in order to become more familiar with the infant's signals and tolerance of stimulation, and parents' modes of responding to the infant. Thereafter, a flexible protocol of procedures matched to infant PMA, familial/ cultural preferences and infant's demonstrated readiness to receive stimulation, will occur as individually tailored MT sessions at bedside:

From 26 to below 32 weeks' PMA, cautious use of sung/toned voice will be the main focus. During MT, the infant may be held in a static manner by the caregiver (eg, skin-to-skin care), resting in his/her isolette with the portal door open, or resting in his/her basinet or bed. MT during skin-to-skin care will be encouraged, if skinto-skin care is part of standard care and the infant tolerates it. The music therapist assesses the infant's needs and behaviour state, and if appropriate for the infant, shows caregivers how to use basic touch and positioning (such as caregiver/therapist's hand lightly and statically placed on infant's chest or back, or hands cupped at infant's head and feet) and/or principles of facilitated tucking and midline alignment to promote physical containment, enable behaviour state regulation and prepare the infant for MT. Parents will hold their infant or provide basic touch and/or positioning, and will receive support or demonstration from the music therapist as needed. Using toning or predominantly wordless singing voice matched to infant breathing patterns, facial expressions and movements, ${ }^{41}{ }^{42}$ while monitoring infant behaviour state shifts and physiological responses, the music therapist demonstrates how to either pacify the infant to promote sleep or bring the infant to a quiet alert state, depending on the infant's needs. Specific pitches used may be matched to the pitch of infant vocalisations, if present, or selected to mask environmental noises, and will aim to be comfortably within the parent's vocal range ${ }^{43}$ When appropriate for the infant, simple melodies or songs of kin ${ }^{31} 43$ modified into an infant-appropriate style are matched to infant breathing rate, gesticulations and facial expressions, and engagement/disengagement cues, so that the music remains infant-directed. Caregivers are encouraged to lead the singing/toning as soon as they feel comfortable, while the music therapist simultaneously provides musical and/or coaching support. MT may occur during breastfeeding or other feeding attempts, if the parents desire it and the infant tolerates it, but MT will not occur during painful or sensorially demanding medical procedures due to the risk of over-stimulation.

From 32 weeks to below 36 PMA, the use of MT centres around cautious use of infant-directed music with expanded sensory experience. This phase applies as soon as the infant demonstrates readiness to receive additional sensory stimulation, but not prior to 32 weeks' PMA. Guidelines from the previous phase apply and are developed on in this phase for infants who are ready for more than pacification. Culturally relevant, caregiver-preferred songs are modified in an improvisatory manner by caregivers to match infant's breathing rate, engagement/disengagement cues, facial expressions and gesticulations, in order to facilitate a musical interplay between caregiver and infant. ${ }^{41} 42$ This interplay generally moves from more pacifying in nature to more dynamic and interactive as the infant reaches 36 
Table 1 Key elements of the music therapy intervention

\begin{tabular}{|c|c|c|}
\hline Phase of intervention & Key elements & Intervention description \\
\hline \multirow[t]{2}{*}{ All phases } & $\begin{array}{l}\text { High parental/caregiver involvement and } \\
\text { resource-oriented }\end{array}$ & $\begin{array}{l}\text { Parents/caregivers are recognised as possessing inherent } \\
\text { resources that can aid their infant and themselves } \\
\text { following premature birth. In all aspects of the MT } \\
\text { intervention, parents are encouraged to take a leading } \\
\text { role, while receiving support from the music therapist. }\end{array}$ \\
\hline & $\begin{array}{l}\text { Therapeutic benefit aimed at infant- } \\
\text { parent dyad/triad }\end{array}$ & $\begin{array}{l}\text { MT promotes infant-parent mutual regulation and includes } \\
\text { psychotherapeutic support of parents. }\end{array}$ \\
\hline
\end{tabular}

Positioning to promote beneficial mutual Infant/parent positioning is considered a vital component regulation for promoting beneficial self-regulation.

Based on needs of infant/parent(s) in the Infant and family needs are assessed at the beginning of moment each intervention phase (during NICU and postdischarge), and during each MT session to assure that use of MT matches needs in the moment (eg, promoting sleep vs encouraging quiet alert interaction).

Parental voice as foundation for infant- Parents' use of their sung (and when necessary, spoken) directed use of song voice along with positioning and physical presence forms the foundation of an attuned response to their infant. Parents' sung voice, facial expressions and vocal expressions vary in response to infant's engagement/ disengagement cues, breathing rate, gesticulations and facial expressions, so that the music remains infantdirected.

Infant medically stable, Cautious use of sung/toned voice in NICU, 26 to below 32 weeks PMA
Predominantly wordless singing voice is matched to infant breathing patterns, facial expressions and movements, and either promotes sleep or a quiet alert state depending on infant's needs.

Incorporation of familiar songs adapted for the premature neonate

Infant medically stable, Cautious use of infant-directed song in NICU, 32 wks to below with expanded sensory experience 36 weeks $\mathrm{PMA}^{*}$

Addition of rhythmic cues and musical phrasing cues to support feeding attempts

Accompanying instruments are used to support the dyad/triad, with complexity matched to infant readiness

Infant medically stable, Expanded engagement in musical in NICU, from 36 weeks exchange

PMA
Parents learn how to adapt songs that are familiar/ preferred so that they are appropriate for the preterm neonate (eg, simplified and/or transformed into lullaby style).

Culturally relevant, caregiver-preferred songs are adapted in the moment to match infant breathing rate, engagement/disengagement cues, facial expressions and gesticulations, to promote musical interplay when appropriate for the infant. The musical interplay may and phrasing to promote rudimentary musical dialogue, eye contact and social responses.

By adapting familiar songs, rhythmic aspects of musical phrasing, and the addition of mildly stimulative vocal sounds can be matched to infant engagement/ disengagement cues to support suck-swallow-breathe coordination and promote mutual regulation.

The music therapist may use acoustic instruments (eg, nylon string acoustic guitar, monochord) to provide single tones or simple accompaniment to support infant/parent, if infant tolerates the added musical stimuli well.

Parents adapt preferred songs so that vocal inflection and musical phrasing reflect infant responses and promote eye contact, vocalisation and rudimentary social interaction, if appropriate for the infant. Parents may also use their positioning while singing to promote auditory localisation, auditory tracking or eye contact. include dynamic touch, and parents' use of vocal inflection 
Table 1 Continued

\begin{tabular}{|c|c|c|}
\hline Phase of intervention & Key elements & Intervention description \\
\hline \multirow[t]{2}{*}{$\begin{array}{l}\text { Postdischarge from } \\
\text { NICU }\end{array}$} & $\begin{array}{l}\text { Based on needs of infant/parent(s) in } \\
\text { the moment and in relation to recent } \\
\text { challenges }\end{array}$ & $\begin{array}{l}\text { Infant and family needs are assessed at the beginning of } \\
\text { each MT session, and particular strengths and challenges } \\
\text { that have arisen in the last } 2 \text { weeks are identified and } \\
\text { targeted (eg, transitioning between behaviour states, } \\
\text { maintaining alertness during feeding, experiencing } \\
\text { rewarding relation). }\end{array}$ \\
\hline & $\begin{array}{l}\text { Identification of musical resources that } \\
\text { may help address current challenges or } \\
\text { build on current strengths }\end{array}$ & $\begin{array}{l}\text { Parents and music therapist brainstorm how music can } \\
\text { be adapted and used for infant/family needs, and parents } \\
\text { have a chance to try out certain approaches with support, } \\
\text { if appropriate. }\end{array}$ \\
\hline
\end{tabular}

*Elements of the prior phase apply to subsequent phases. An infant remains in the previous phase (despite PMA) until ready to receive more expanded stimulation. The combination of elements used within each phase will depend on the infant's needs in the moment. MT, music therapy; NICU, neonatal intensive care unit; PMA, postmenstrual age.

weeks' PMA. Musical interplay may include gentle dynamic touch to back of infant's head or along infant's back, depending on the infant's tolerance. The musical interplay can be used to support the infant in a quiet alert state, if appropriate for the infant. Vocal inflection may be used to promote eye opening, eye contact and responses to social cues (eg, smiling, cooing). The infant-directed improvised interaction will be paced and modified according to infant responses and engagement cues, and will be reversed or paused in response to disengagement cues. Conversely, for infants who have difficultly rousing for meal times, sessions can begin as described in 'cautious use of sung/toned voice', with caregiver/therapist hand placed lightly and statically on infant's chest or back, or both hands/wrists lightly and statically cradling the sides of the infant and supporting the infant to align at midline. If the infant tolerates infant-directed singing in these positions, the caregiver/ therapist can progress to include gentle rhythmic cues via light touch to coax the infant into a quiet alert state. If MT is used during breastfeeding attempts, rhythmic aspects of musical phrasing, ${ }^{53}$ and the addition of slightly stimulative vocal sounds can be matched to infant engagement/disengagement cues to support suck-swallow-breathe coordination and promote infant-parent mutual regulation. ${ }^{54}$ The music therapist may use simple acoustic instruments (eg, nylon string acoustic guitar, monochord) with single tones or simple accompaniment to accompany infant/caregiver during this phase, if the infant tolerates such additional stimulation without disengagement cues or physiological signs of overstimulation.

From 36 weeks' PMA, MT will focus on expanded engagement in musical exchange. This phase applies when the infant demonstrates readiness to engage in increasingly more interactive levels of musical exchange. Caregivers interact musically with the infant, using vocal inflection and musical phrasing to encourage the infant to achieve a quiet, alert state and engage in eye contact, vocalisation and rudimentary social interaction, if appropriate for the infant. Depending on infant readiness, caregivers may use their voices and physical positioning to promote auditory localisation, auditory tracking and eye contact; and their fingers and hands to promote reaching, grasping, and mouthing. The therapist will encourage the caregiver to adapt preferred lullabies and children's songs or to modify songs of kin ${ }^{31} 43$ in order to encourage musical dialogue with the infant, and the therapist may provide basic harmonic or melodic accompaniment as support.

\section{Standard care during NICU}

This will vary across countries, but will typically include necessary medical care and a limited amount of interventions to reduce stress among infants and to inform and promote safety in parents. ${ }^{55}$ Components of standard care will be recorded at each site.

\section{MT postdischarge from hospital}

Infant-parent dyads will be offered $45 \mathrm{~min}$ individual MT sessions, 7 times distributed across the first 6 months after discharge ( 2 times/month for the first month, and 1 time/month thereafter). These will occur at home, municipal child health centres or follow-up clinics and comprise: (1) Verbal greeting and brief discussion of infant's progress and parents' related coping ( $5 \mathrm{~min})$. (2) Musical greeting of infant and caregiver, encouraging caregiver to participate $(5 \mathrm{~min})$. (3) Engagement in musical exchange following the procedures described in the 'from 36 weeks' category above and incorporating adapted play songs, with therapist modelling musical engagement ( 10 min). (4) Discussion of current infant/ parent challenges and strategies for using musical interactions to address these needs, with therapist modelling 
musical techniques to promote self-regulation or to facilitate musical interaction, depending on needs identified by the caregiver $(\sim 10 \mathrm{~min})$. (5) Caregiver demonstration of techniques discussed during session ( $10 \mathrm{~min})$. (6) Musical closure and reminder of planned timing for next visit ( $\sim 5 \mathrm{~min})$. Any siblings may be involved if desired by the caregiver, and sessions will incorporate musical instruments and resources present in the home, if appropriate for the infant. Subsequent sessions will follow a similar sequence, adjusted to infant developmental level and ongoing needs. The therapist will work in close dialogue with those providing standard aftercare procedures, when possible.

\section{Standard care postdischarge from hospital}

This includes follow-up visits and preventive interventions in primary or specialist healthcare as needed. Preventive interventions during the first year of life are focused on growth/eating/nourishment as well as psychomotor/ sensory development and also include a focus on families and bonding. ${ }^{55}$ Standard care may include other early intervention methods of care with the exception of other MT approaches.

\section{Training in study procedures}

The site investigator at each site will maintain responsibility for assuring that the trial procedures are implemented as intended. Site investigators and music therapists received initial training on study procedures during an in-person, 3-day training in June 2018. In addition, site investigators, music therapists and other site research personnel received online follow-up training from core team members prior to the commencement of the study at their site. Music therapists will receive additional monitoring and feedback on their first two MT sessions from NICU phase and first session from postdischarge phase, using review of audio/video recordings obtained for subsequent evaluation of treatment fidelity. The core team will maintain dialogue with each site at least monthly during implementation of the study to assure adherence to the protocol, answer questions and help problem-solve possible challenges.

\section{Treatment guidelines for MT}

MT during NICU and postdischarge phases are conducted in accordance with treatment guidelines established for this study. Guidelines are grounded in current evidencebased practices, experiences from the Norwegian feasibility study and feedback from our UAG and Scientific Advisory Committee (SAC). These treatment guidelines are used in training the music therapists involved in the study, and help promote consistency of treatment implementation across study sites. The treatment guidelines articulate guiding principles underlying the intervention, delineate the level of variation that is acceptable and provide illustrative examples of approaches. Implementation of the guidelines allows for individual tailoring to meet the needs of the infant and/or parents, and presumes a music therapist trained specifically in the use of MT for premature infants and their families. Treatment guidelines will be available as a separate paper.

\section{Assessment of treatment fidelity}

We will assess treatment fidelity in terms of treatment delivery and treatment receipt. A predetermined selection of audio recordings from NICU MT sessions and video recordings from postdischarge MT sessions will be assessed for adherence to core elements of the intervention. Extent of adherence during treatment delivery will be assessed by music therapists reviewing recordings of their sessions and completing self-ratings, and external raters reviewing the same recordings to determine extent of adherence. Inter-rater reliability between the external raters will be evaluated, and degree of correspondence between the external and therapist self-ratings will be determined, as appropriate. We will evaluate fidelity in regard to treatment receipt by parental self-rating at the 6-month CA timepoint.

\section{Outcomes}

Unless indicated otherwise, all outcomes will be assessed at baseline; 1-2 days before discharge to home; at 6-month CA (end of MT; primary endpoint); and at 12 months of CA (figures 1 and 2). All applicable outcomes (ie, all that are scale-based) will be calculated as final values, but where baseline scores are available they will be included in the statistical models (see below). Data collectors and assessors will be trained in assessment procedures and blinded to participant allocation; success of blinding will be verified. For all self-report instruments we will use official translations, or if not available, we will translate according to recommended procedures with translations, back-translation and discussions before the final version is completed.$^{56}$ Psychometric properties of the instruments will be assessed across sites.

\section{Primary outcome}

Bonding between primary caregiver and infant at 6-month CA will be measured using the $\mathrm{PBQ}^{57}{ }^{58}$; the same outcome will also be assessed at baseline, discharge, and at 12-month CA. The PBQ is a parent-rated screening instrument for disorders of the early mother-infant relationship consisting of 25 statements on a 6-point Likert scale (each $0-5$; sum score ranging from 0 to 125 ; high=problematic), addressing problems in the mother-infant relationship based on weakened bonding; rejection and anger; anxiety about care; and risk of abuse. ${ }^{59}$ The scale is validated and widely used in clinical practice and research. ${ }^{39}$ It has been translated and tested in several languages and cultures ${ }^{58}$ and has been found to be reliable in mothers with or without depression or bonding problems. ${ }^{59}$ Results from feasibility testing and user feedback in Norway confirmed the acceptability and relevance of the $\mathrm{PBQ}$, also in comparison to alternative but less widespread measures that were considered. The 
scale covers both the more common and the rare but important problems that parents and infants can have.

\section{Secondary infant outcomes will be as follows}

1. The most important among the secondary outcomes is child development at 24 months of CA, as indicated by the Bayley Scales of Infant and Toddler Development, third edition (Bayley-III). The Bayley-III is considered the gold standard for assessing development in young children up to 42 months. ${ }^{60}$ At 24 months, the tool is used routinely in many countries for extremely premature infants. Assessors will be trained and reliability-tested before conducting the assessments for this study, also in cases where the assessments are part of routine procedures, when possible. The three main composite scores of the Bayley-III are cognitive, language (receptive and expressive) and motor (fine and gross motor) development; each of the scales is standardised with a population mean of 100 (SD 15), with higher scores indicating better development. No total score covering all domains is available, but the most important areas for the present study will be language and cognition. The Bayley-III has US norms and has been used extensively for developmental assessments for premature infants (eg, Luttikhuizen dos Santos $\left.e t a b^{61}\right)$.

2. Infant development at 6-month and 12-month CA will be assessed by the Ages and Stages Questionnaire, third edition (ASQ-3). ${ }^{62}{ }^{63}$ The ASQ-3 is an age-specific parent-reported screening questionnaire consisting of 30 items covering five developmental domains with five subscales: communication, fine-motor, gross-motor, problem-solving and personal-social (possible range 0-60) and a total score (possible range 0-300), with higher scores indicating better development.

3. Infant socioemotional development will be measured by the Ages and Stages Questionnaire: Social-Emotional, second edition (ASQ:SE-2) at 6 and 12 months of CA. This is a parent-completed questionnaire with 19 or 22 Likert-scaled items (each 0-5-10), plus additional items for whether an item is of concern to the parent (each $0-5$ ), resulting in a score ranging from 0 to 285 or 0 to 300 , at 6 and 12 months, respectively. Lower scores indicate better socioemotional development. $^{6465}$

4. Rehospitalisation during the first year of life (excluding outpatient visits), based on electronic health records or parent reports. This will be calculated as the time from initial discharge until first rehospitalisation.

\section{Secondary parental outcomes include the following}

1. Maternal depressive symptoms will be assessed with the Edinburgh Postnatal Depression Scale (EPDS) at baseline, 1-2 days prior to discharge, and at 6 and 12 months of infant CA. The 10 -item validated self-report instrument assesses mothers' postpartum depressive symptoms, excluding somatic symptoms of depression that are common in new mothers (such as loss of energy, feeling tired, changes in appetite and sexual drive). ${ }^{66}$ Scores can range from 0 to 30 , with high scores indicating more depressive symptoms.

2. Level of anxiety (both mothers and fathers) will be assessed with the Generalised Anxiety Disorder Assessment (GAD-7) at baseline, 1-2 days prior to discharge, and at 6 and 12 months of infant CA. The self-report 7-item questionnaire serves as a screening tool and severity measure for generalised anxiety disorder. ${ }^{67}$ Scores can range from 0 to 21, with higher scores indicating higher anxiety.

3. Level of parental stress (both mothers and fathers) will be assessed with the Parental Stress Scale (PSS) at 6 and 12 months of infant CA. This is a self-report 18item questionnaire that assesses stress associated with parenting. ${ }^{68}$ Scores can range from 18 to 90 , with higher scores indicating higher stress.

\section{Other measures}

Specific medical and social factors relevant for subgroup analyses will include parents' socioeconomic status (collected at baseline), infants' hearing status (collected at discharge), frequency of skin-to-skin care during NICU, and experience of breastfeeding prior to 6-month CA. Additional open-ended questions will address other potential factors that might impact infant responsiveness (which may include, eg, intraventricular haemorrhage, metabolic disturbances, or genetic disorder among infants, and known mental health or substance use problems of parents). These will be drawn from hospital records and may be supplemented by parent self-reports.

\section{Cost-effectiveness}

We will explore and collect data necessary for analysing cost-effectiveness both from a health services perspective (ie, costs of all treatments incurred within the health sector including the costs of MT and standard care) and from the broader societal perspective (ie, including all treatments incurred by the health sector and other indirect costs such as productivity losses and out-of-pocket expenses incurred by the parents). If clinical effectiveness is found, we will apply for separate funding to explore the additional cost to achieve a unit improvement in parent-infant bonding and explore the likelihood MT will be good value for money. This will be done through incremental cost-effectiveness ratios and willingness to pay analyses. Otherwise, these data will be used to describe the context in which the treatment took place.

\section{Adverse events}

All significant harms as well as unintended effects for each group will be collected and described by the site investigator, and reported to the core team. All adverse events that site investigators suspect may be related to MT and all serious adverse events will be reported to the Data and Safety Monitoring Committee (DSMC) by the core team. A special form to report trial-related adverse events has been developed and distributed. 
Sample size and power calculation for the main comparison

No previous RCT examined effects of MT on the PBQ; two small RCTs ${ }^{38}$ and a recent non-randomised study ${ }^{46}$ examined mother-infant bonding, but provided insufficient data for meta-analysis. Studies using the PBQ with other interventions found effects ranging widely from around 0.25 to 9.0 , with SDs ranging from 4 to $12 .{ }^{11} 6970$ Assuming a difference of 4 points on the $\mathrm{PBQ}(\mathrm{SD}=8)$ as a minimal clinically important difference for this study, power of $80 \%$ will be achieved for each main effect (each tested on a two-sided $2.5 \%$ significance level, ie, $5 \%$ with Bonferroni correction for two tests) with a sample size of 155. Taking into account some clustering by country (ICC $0.01 ; 5$ countries), this is increased to 203. To allow for $20 \%$ attrition, we will aim to include 250 infants $(\sim 50$ in each country) and their parents. This sample size will also ensure power for testing proportion differences of about $15 \%$ (eg, binary analysis of problematic bonding; rehospitalisation).

Special considerations are needed for multiple pregnancies (twins, triplets and so on), which account for about $2 \%$ of all pregnancies in the Hordaland region of Norway, ${ }^{71}$ but are more commonly encountered in NICUs due to elevated risk of preterm birth. Although including all siblings would serve to increase the sample size, it may lower the resulting power due to cluster effects. Therefore, we will only formally enrol and analyse the first-born sibling of each multiple pregnancy, although all multiples can receive the allocated intervention (see the Eligibility criteria section).

\section{Recruitment}

Recruitment at the first site commenced in July 2018, and completion of recruitment is planned for the end of 2019. Enrolment will be completed in the NICU by a trained member of the unit staff according to the eligibility criteria. The staff person will be responsible for first contact with caregivers, providing an opportunity for informed consent. We estimate that an 18-month period for recruitment will be sufficient to achieve the targeted sample size. Each site will be encouraged to recruit $~ 50$ infants and their parents; therefore, slow recruitment at some sites will be tolerable. Recruitment rates will be monitored carefully. Local and national partners responsible for primary care provision will serve as contact points after discharge and will be actively involved, wherever possible. Once an infant is randomised, the site investigator will make every reasonable effort to follow the infant and the parent/caregiver for the entire study period of 24 months. The site investigator will be responsible for developing local standard operating procedures for the site, in order to facilitate implementation of all aspects of the study. In addition, site investigators will develop postrecruitment retention strategies to promote retention (eg, development of a newsletter directed to participants).

\section{Randomisation and allocation concealment}

After site-specific informed consent and baseline assessment, participants will be randomly assigned to MT during
NICU or standard care using a computer-generated randomisation list, with ratio 1:1, in blocks with sizes of 2 or 4 varying randomly, stratified by site using an online system. The random allocation sequence will be generated and administered by people with no involvement in the clinical work to ensure allocation concealment. One potential issue with providing MT in shared NICU rooms is contamination. Parents may pick up strategies from other parents in the same room (or, likely to a lesser extent, from other parents or staff on the ward). We will assess contamination through the following questions at the end of each parent's participation in the study: 'How often did you sing or play music with or for your child (daily/weekly/occasionally/ never)? If so, in what way (please describe)? Did you learn from another parent in the NICU about using music (yes, please describe/no)?'

Before discharge to home, participants will be randomised a second time to postdischarge MT or standard care in a 1:1 ratio, using the same procedures. This two-step randomisation was chosen as the best way to create balance among those remaining in the study for the postdischarge phase, and to avoid differential drop-out in the NICU phase due to expectations for the postdischarge phase. We will assess potential contamination in the postdischarge phase by asking participants if they engaged in MT with their infant outside of the study during the first year of the infant's life, and if so, the frequency and nature of such engagement.

\section{Blinding}

Due to the nature of the intervention neither participants nor staff can be blinded to allocation, but are reminded not to disclose the participant's allocation status to outcome assessors. All outcomes that are not self-reports will be conducted by an assessor blind to treatment allocation, and success of blinding will be tested by asking assessors.

\section{Data analyses}

Baseline analysis

Sociodemographic and clinical baseline properties for the groups will be characterised by descriptive methods (mean (SD), median (range), n (\%)).

\section{Primary analysis}

We will use an intention-to-treat approach using all available data from all participants as randomised, regardless of the intervention actually received. Multiple imputation will be used for missing data, if applicable. ${ }^{72}$

Effects will be examined by testing the randomised groups for differences in the primary endpoint ( $t$-test or Mann-Whitney; change in PBQ scores to 6-month CA), as well as by fitting linear mixed models. In the first modelling step, we will assess the effect of the first randomisation (figure 1) using the following model:

$$
P B Q_{i t}=\beta_{0}+\beta_{1} \text { time }_{t}+\beta_{2} g_{i}+\beta_{12} \text { time }_{t} g r_{i}+U_{i}+\varepsilon_{i t},
$$

where $P B Q_{i t}$ denotes the $P B Q$ score for participant $i$ at measurement $t$, time $_{t}$ the time from baseline at measurement $t$ and $g r_{i}$ the randomisation group of participant 
i. $\beta_{0}, \beta_{1}, \beta_{2}$ and $\beta_{12}$ are the coefficients estimated in the model, $U_{i}$ is an individual random term per participant and $\varepsilon_{i t}$ an independent, identically distributed error term. In the second step, we refine the model to take into account both randomisation steps, leading to the extended model:

$$
\begin{aligned}
P B Q_{i t}= & \beta_{0}+\beta_{1} \text { time }_{t}+\beta_{2} g r R 1_{i}+\beta_{12} \text { time }_{t} g r R 1_{i}+ \\
& \beta_{3} g{ }^{+} 2_{i}+\beta_{13} \text { time }_{t} g r R 2_{i}+U+\varepsilon_{i t},
\end{aligned}
$$

with the same notation as above and $g r R 1_{i}$ and $g r R 2_{i}$ denoting the groups from the first and second randomisation, respectively. In these models $\beta_{1}$ describes a group-independent time effect, $\beta_{2}$ and $\beta_{3}$ a time-independent group effect, and $\beta_{12}$ and $\beta_{13}$ the time-dependent change of the group effect. We will investigate both linear and simple contrasts (comparing each time point with the baseline) in time to assess the treatment effect.

\section{Secondary analyses}

The same analyses will be done for the secondary outcomes; using an analogous generalised linear mixed model for binary outcomes. Perprotocol analyses will include those who received at least 6 MT sessions in the NICU and at least 5 MT sessions postdischarge, respectively; these are considered the minimum numbers for the intervention to be successful.

\section{Subgroup analyses}

Additionally, we will conduct exploratory statistical analyses for prespecified subgroups: sex; GA at birth $(<28$, 28 to $<32,32$ to $<35$ weeks); hearing status at discharge (normal vs abnormal); bonding at baseline (PBQ impaired bonding score $\geq 11$ vs $<11$ ) ; parental socioeconomic status (poor vs other) ${ }^{73}$; average parental skin-toskin care during NICU ( 4 or more days per week vs fewer than 4 days per week); duration of breastfeeding prior to 6-month CA (none, fewer than 3 months, 3 months or greater); perceived emotional closeness during breastfeeding (consistently, sometimes, rarely); as well as qualitative/mixed-methods analyses of process data to maximise learning.

\section{Data management and collection methods}

In order to maintain participant confidentiality all information will be stored with ID code numbers. The ID code numbers will be unrelated to participants' identifiers, except in a central file with the participants' contact details. All data will be stored on an electronic database management system located on a secure server with password-controlled access provided for research data collectors.

To ensure data quality, a trial database will be set up and maintained using a safe server (Uni Research Health Data Storage, UHEADS; now maintained by NORCE) and OpenClinica software. UHEADS is a system for safely storing health research data that accommodates the safe upload, storage and retrieval of any sensitive research data. OpenClinica is a web-based system for electronic data capture and clinical data management for multicentre clinical trials, which conforms to relevant international standards for health research. NORCE Norwegian Research Centre AS runs an open source version of OpenClinica. A manual for using OpenClinica in this study has been created and distributed to collaborators. All records that contain names or other personal identifiers, such as informed consent forms, will be stored separately from study records identified by the ID code number. All study-related information on paper, including questionnaires, and administrative forms will be securely stored in locked file cabinets in areas with limited access.

\section{Data availability statement}

After completion of the study period, de-identified individual participant data of the main variables will be stored on a publicly available repository to ensure replicability and transparency, namely Norwegian Centre for Research Data (NSD).

\section{Monitoring}

The site investigators will practice entering data so that the core team can confirm that site investigators are proficient in all aspects of data entry, query response and communication with the team at each site. Uploaded data will be regularly checked by a core team researcher in order to ensure good quality data. Monitoring will follow a risk-based approach, with site investigators and core team members sharing the responsibility for quality and completeness of data. Core team members will conduct monitoring of source documents via scan of randomly chosen questionnaires at all enrolling LongSTEP sites, and will conduct on-site monitoring visits as needed. Through these on-site visits we will audit the overall quality and completeness of the data, examine source documents and interview coordinators to determine whether data reported in OpenClinica are complete and accurate, and whether the clinical site has complied with the requirements of the protocol. If a problem is identified, the core team will assist the site investigator in resolving the issues.

\section{Oversight, auditing and advisory committees \\ Data and Safety Monitoring Committee}

An independent panel of three clinicians and methodologists with relevant expertise but no direct involvement in the trial has been established to ensure research integrity and safety of participants in the trial. After an initial protocol review meeting, the DSMC will check the general progress of the clinical trial including recruitment and completeness of follow-up; safety; and any other unforeseen events. To this end, the DSMC will receive updated reports from the project statistician, which may be unblinded with respect to intervention arms. Other project team members will not have access to such unblinded reports and will not attend the closed part of the DSMC meetings where such content is discussed. No interim efficacy analysis is planned for this trial. 


\section{Trial Steering Committee}

The Trial Steering Committee will consist of one representative from each recruiting site, two user representatives, and two representatives from the core team. It will monitor trial progress, will receive recommendations from the DSMC, and will be involved in all decisions about protocol amendments or other changes in the trial.

\section{User Advisory Group}

An advisory group consisting of parents of premature infants in Norway has been established for this study. This group is important for eliciting user feedback and ensuring relevance of the study, including any protocol amendments, as well as helping to ensure efficient recruitment and dissemination strategies. Similar involvement of users in other countries will be encouraged.

\section{Scientific Advisory Committee}

An international group of clinicians and researchers with relevant clinical expertise was established during project development and will continue to advise on various aspects related to development and implementation of the study. The panel includes experts in areas such as MT, neonatology and psychology.

\section{Research ethics approval}

The project as a whole received ethics approval from The Regional Committees for Medical and Health Research Ethics (2018/994/REK Nord, date of approval: 03 July 2018). In addition, each participating site must secure local ethical approval prior to commencing recruitment. Site-specific written informed consent will be obtained postnatally following routine practices in the NICU. A trained staff of the trial will inform parents/caregivers about the study and provide an opportunity for informed consent. The parent/caregiver will receive written and oral explanation of the proposed research project, including information regarding the research project, the aims of the project, the duration of the participant's involvement, the expected benefits to the participant and others, the nature of the intervention and the procedures involved in participation. It will be emphasised that enrolment in the study is voluntary, that parents/caregivers can withdraw at any time from all or part of the study, and that any decision they take in this respect will have no bearing on the medical care received. Additionally, it will be highlighted that information generated by the study will be published, but that no details will be divulged from which the participant could be identified. Primary caregivers will be informed of whom to contact in an emergency, and the staff will be available to answer questions.

\section{Dissemination}

Dissemination of the findings will apply to local, national and international levels. Aspects related to the feasibility of implementing MT in the hospital and in primary care and region-specific and culture-specific aspects of treatment implementation will be submitted to corresponding specialised interdisciplinary journals. Main results of the trial concerning clinical effectiveness will be submitted to a leading medical journal. Additional results of exploratory statistical analyses and qualitative/mixed-methods analyses of process data will be published in specialised international journals. Beyond journal publications, we will disseminate results through presentations at national and international conferences. Popular dissemination, particularly to users, their families, user organisations, health services, and the general public will be achieved via a periodic newsletter, educational brochures and/or popular press articles.

\section{DISCUSSION}

Controlled research on the impact of MT for premature infants and their caregivers demonstrates short-term improvements in infant physiologic and behaviour states, feeding behaviour and length of hospital stay, as well as decreases in parental distress and anxiety. ${ }^{31} 3438$ LongSTEP fills a critical gap in knowledge by providing MT over a longer term from hospitalisation through the first 6 months of life, and by assessing long-term outcomes of MT for both infant and parents during the first 2 years of the infant's life.

Since MT for premature infants and their caregivers is an emerging area of practice and research within Scandinavia, this study aims to integrate best available evidence from international developments with standards of practice existing in specific national healthcare environments, to create a model of care that is culturally relevant for social support societies. LongSTEP consists of MT with a high level of parental engagement during the course of NICU hospitalisation and a support-to-parent and consult-to-parent model of MT for the first 6 months following discharge. The model enables parents to assume a central role in the care of their infant, with the music therapist providing support and coaching to help parents to use their innate resources to promote developmentally appropriate mutual regulation of the parent/ infant dyad, improve quality of interaction and enable healthy bonding during this vulnerable time. The longterm aim is to assure healthy infant-parent relation and improve child developmental outcomes and promote parental psychological well-being over the first 2 years of the infant's life.

The pragmatic nature of this trial and its high clinical applicability are reflected in several dimensions, as described in the pragmatic-explanatory continuum indicator summary. ${ }^{74}$ Eligibility criteria are designed to be as inclusive as possible to promote applicability of the evidence with respect to a broad population. Broad inclusion criteria ensure enrolment of participants with heterogeneous characteristics similar to those seen by clinicians in daily practice, and exclusion criteria are narrow and exist only to ensure infant safety. Another pragmatic dimension of this trial is flexible MT delivered by a qualified music therapist. MT is tailored to infants over a broad range of PMA and also 
incorporates family and cultural preferences. Additionally, we do not restrict if or how cointerventions will be delivered. Participants in all arms will receive standard care. Analysis of primary outcome will include all individuals regardless of compliance in order to test if the intervention works under usual conditions, with all the noise inherent therein.

As a limitation it should be noted that our primary outcome measure is a self-report tool that is completed by parents who know which intervention they and their infant received. It is an inherent challenge within MT research that we cannot blind participants and providers, as they are aware that they have received MT. We believe that mothers' subjective perception of their bond with their infant is an important phenomenon to evaluate and target with supportive intervention. We hope to offset the use of a self-report primary outcome completed by non-blinded participants by including the Bayley-III to provide an observational evaluation of infant development completed by independent, blinded outcome assessors.

Clinical benefits as well as resulting societal benefits related to disability prevention and economic outcomes are likely to occur over a longer term. To address this, we will also include the possibility in the consent forms to collect later follow-up data, but these will be beyond the present project. In summary, this pragmatic trial will determine the effects of MT under usual conditions, and the design as a multicentre trial will further ensure wide generalisability.

The project's social impact includes filling gaps in knowledge regarding the long-term impact of MT with preterm infants/caregivers, including highly user-relevant outcomes. The high applicability of this trial will facilitate ongoing development of evidence-based care for preterm infants. Opportunities for improved training of music therapists and effective implementation of MT in neonatal care will apply both to countries where MT in this area is new and to countries that already have a more established foundation. Specifically, LongSTEP will make a unique contribution to continuity of care by bridging intervention from NICU hospitalisation to municipal and home settings postdischarge. In addition, LongSTEP fosters interdisciplinary collaboration between specialist and primary/municipal healthcare services (psychologists, nurses, physicians, music therapists).

In conclusion, LongSTEP builds off of evidence-based practice in neonatal MT, which supports the use of infant-specific MT tailored to developmental level and provided in conjunction with primary caregivers. The project will advance previous knowledge by suggesting a pathway by which MT can promote infant development while also improving parental psychological outcomes. Coaching parents in the use of music may help improve infant regulation and decrease parental stress during parent/infant interactions, which in turn may promote bonding and contribute to better infant developmental and parental psychological outcomes.
Author affiliations

${ }^{1}$ GAMUT - The Grieg Academy Music Therapy Research Centre, The Grieg Academy - Department of Music, University of Bergen, Bergen, Norway

${ }^{2}$ GAMUT - The Grieg Academy Music Therapy Research Centre, NORCE Norwegian Research Centre AS, Bergen, Norway

${ }^{3}$ Institute of Psychology, Uniwersytet Gdanski, Gdansk, Poland

${ }^{4}$ Department of Psychosocial Science, University of Bergen, Bergen, Norway

${ }^{5}$ Regional Center for Child and Youth Mental Health and Child Welfare, NORCE

Norwegian Research Centre AS, Bergen, Norway

${ }^{6}$ King's Health Economics, King's College London, London, UK

${ }^{7}$ Department of Patient and Family Care, Hospital Universitario Fundacion Santa Fe de Bogotá, Bogotá, Colombia

${ }^{8}$ SONO - Centro de Musicoterapia, Bogota, Colombia

${ }^{9}$ Neonatal Department, Meir Medical Center, Kfar Saba, Israel

${ }^{10}$ Sackler School of Medicine, Tel Aviv University, Tel Aviv, Israel

${ }^{11}$ Department of Pediatrics, Haukeland University Hospital, Bergen, Norway

Acknowledgements Hanne Cecilie Braarud, members of our User Advisory Group (Trude 0s, Anette Christine Røsdal, Signe Hjelen Stige) and members of the Scientific Advisory Committee (Deanna Hanson-Abromeit, Friederike Haslbeck, Małgorzata Lipowska, Joanne V Loewy, Renate Nussberger, Helen Shoemark, Alexandra Ullsten) provided valuable comments on earlier versions of this protocol. We thank Jo Dugstad Wake for help in setting up the clinical data management system for the trial.

Contributors $Ł B, C G h$ and $C G$ conceived the study, developed the study design and drafted the manuscript. JA developed the study design, provided statistical expertise in clinical trial design and is conducting the primary statistical analysis. IK, MH, RR, ME, SA, BJV and TSG contributed to the study design, with IK and MH contributing in particular the definition of outcomes. All authors contributed to refinement of the study protocol and approved the final manuscript.

Funding The project has been funded by the Research Council of Norway (RCN, project number 273534), under the programme High-quality and Reliable Diagnostics, Treatment and Rehabilitation (BEHANDLING). Additional funding was provided by the Faculty of Fine Art, Music, and Design (KMD) at the University of Bergen, and the POLYFON Knowledge Cluster for Music Therapy. The funders of the study had no role in design and will not have a role during conduct of the study; collection, management, analysis and interpretation of the data; and preparation, review or approval of the manuscript or the decision to submit for publication.

Competing interests $\measuredangle B$, CGh and CGo conceived the study, developed the study design, and drafted the manuscript. JA developed the study design, provided statistical expertise in clinical trial design and is conducting the primary statistical analysis. IK, MH, RR, ME, SA, BV and TG contributed to the study design, with IK and $\mathrm{MH}$ contributing in particular the definition of outcomes. All authors contributed to refinement of the study protocol and approved the final manuscript.

Patient consent for publication Not required.

Ethics approval The Regional Committees for Medical and Health Research Ethics (2018/994/REK Nord, date of approval: 03 July 2018) approved of the main study. A protocol for feasibility trials in Norway and Poland was approved by the Regional Committees for Medical and Health Research Ethics (2017/2249/REK Nord, date of approval: 05 December 2017) and the Research Ethics Board at the University of Gdańsk (no 3/2018, date of approval: 12 April 2018), respectively.

Provenance and peer review Not commissioned; externally peer reviewed.

Open access This is an open access article distributed in accordance with the Creative Commons Attribution Non Commercial (CC BY-NC 4.0) license, which permits others to distribute, remix, adapt, build upon this work non-commercially, and license their derivative works on different terms, provided the original work is properly cited, appropriate credit is given, any changes made indicated, and the use is non-commercial. See: http://creativecommons.org/licenses/by-nc/4.0/.

\section{REFERENCES}

1. Blencowe H, Cousens S, Chou D, et al. Born too soon: the global epidemiology of 15 million preterm births. Reprod Health 2013;10(Suppl 1):S2.

2. Blencowe $\mathrm{H}$, Cousens $\mathrm{S}$, Oestergaard $\mathrm{MZ}$, et al. National, regional, and worldwide estimates of preterm birth rates in the year 2010 with time trends since 1990 for selected countries: a systematic analysis and implications. The Lancet 2012;379:2162-72. 
3. Liu L, Johnson HL, Cousens S, et al. Global, regional, and national causes of child mortality: an updated systematic analysis for 2010 with time trends since 2000. The Lancet 2012;379:2151-61.

4. Twilhaar ES, Wade RM, de Kieviet JF, et al. Cognitive outcomes of children born extremely or very preterm since the 1990s and associated risk factors. JAMA Pediatr 2018;172:361-7.

5. Fevang SKE, Hysing M, Markestad T, et al. Mental health in children born extremely preterm without severe neurodevelopmental disabilities. Pediatrics 2016;137:e20153002-13.

6. Zwicker JG, Harris SR. Quality of life of formerly preterm and very low birth weight infants from preschool age to adulthood: a systematic review. Pediatrics 2008;121:e366-76.

7. Underwood MA, Danielsen B, Gilbert WM. Cost, causes and rates of rehospitalization of preterm infants. J Perinatol 2007;27:614-9.

8. Singer LTet al. Maternal psychological distress and parenting stress after the birth of a very low-birth-weight infant. JAMA 1999;281:799-805.

9. Jotzo M, Poets CF. Helping parents cope with the trauma of premature birth: an evaluation of a trauma-preventive psychological intervention. Pediatrics 2005;115:915-9.

10. Bieleninik $Ł$, Bidzan M, Salwach D. The premature birth trauma and the parents' quality of life in the light of the S.Allen and A. Michalos' models. In: Wellness and success $O$. G. NeuroCentrum: Lublin, 2009: 39-52.

11. Hoffenkamp HN, Tooten A, Hall RAS, et al. Effectiveness of hospitalbased video interaction guidance on parental interactive behavior, bonding, and stress after preterm birth: a randomized controlled trial. J Consult Clin Psychol 2015;83:416-29.

12. Bieleninik $Ł$. Dzieci urodzone przedwcześnie w percepcji matek (Mothers' perception of prematurely born children in the period of the early childhood. Gansk: Harmionia Universalis, 2012.

13. Bieleninik $七$, Gold $C$. Early intervention for premature infants in neonata intensive care unit. Acta Neuropsychologica 2014;12:185-203.

14. Sloan K, Rowe J, Jones L. Stress and coping in fathers following the birth of a preterm infant. J Neonatal Nurs 2008;14:108-15.

15. Lee T-Y, Wang M-M, Lin K-C, et al. The effectiveness of early intervention on paternal stress for fathers of premature infants admitted to a neonatal intensive care unit. J Adv Nurs 2013;69:1085-95

16. Bidzan Met al. Bond with a child in the prenatal period in case of prematurely born children. In: Turowski K, ed. Wellness and success. NeuroCentrum: Lublin, 2009: 35-54.

17. Bowlby J. Attachment and loss: Vol 1. London: Pimlico, 1997.

18. Ainsworth MDSet al. Patterns of attachment: a psychological study of the strange situation. Erlbaum: Hillsdale NJ, 1978.

19. Korja R, Savonlahti E, Ahlqvist-Björkroth S, et al. Maternal depression is associated with mother-infant interaction in preterm infants. Acta Paediatr 2008;97:724-30.

20. Muller-Nix C, Forcada-Guex M, Pierrehumbert B, et al. Prematurity, maternal stress and mother-child interactions. Early Hum Dev 2004;79:145-58.

21. Wolke D, Eryigit-Madzwamuse S, Gutbrod T. Very preterm/very low birthweight infants' attachment: infant and maternal characteristics. Arch Dis Child Fetal Neonatal Ed 2014;99:F70-F75.

22. Fegran L, Helseth S, Fagermoen MS. A comparison of mothers' and fathers' experiences of the attachment process in a neonatal intensive care unit. J Clin Nurs 2008:17:810-6.

23. Olsson E, Eriksson M, Anderzén-Carlsson A. Skin-to-skin contact facilitates more equal parenthood - a qualitative study from fathers' perspective. J Pediatr Nurs 2017;34:e2-9.

24. Korja R, Latva R, Lehtonen L. The effects of preterm birth on mother infant interaction and attachment during the infant's first two years. Acta Obstet Gynecol Scand 2012;91:164-73.

25. Ghetti CM. Pediatric intensive care. In: Bradt J, ed. Guidelines for music therapy practice in pediatric care. Barcelona Publishers: Gilsum, NH, 2013: 152-204.

26. Haslbeck FB. Music therapy for premature infants and their parents: an integrative review. Nord J Music Ther 2012;21:203-26.

27. Standley JM, Walworth D. Music therapy with premature infants: research and developmental interventions. 2 edn. Silver Spring, MD: American Music Therapy Association, 2010.

28. Whipple J. The effect of parent training in music and multimodal stimulation on Parent-Neonate interactions in the neonatal intensive care unit. J Music Ther 2000;37:250-68.

29. Mondanaro JF, Ettenberger M, Park L. Mars rising: music therapy and the increasing presence of fathers in the NICU. Music and Medicine 2016;8:96-107.

30. Hartling L, Shaik MS, Tjosvold L, et al. Music for medical indications in the neonatal period: a systematic review of randomised controlled trials. Arch Dis Child Fetal Neonatal Ed 2009;94:F349-F354.
31. Loewy J, Stewart K, Dassler A-M, et al. The effects of music therapy on vital signs, feeding, and sleep in premature infants. Pediatrics 2013;131:902-18.

32. Standley JM. Music therapy for the neonate. Newborn and Infant Nursing Reviews 2001;1:211-6.

33. Standley JM. A meta-analysis of the efficacy of music therapy for premature infants. J Pediatr Nurs 2002;17:107-13.

34. Standley J. Music therapy research in the NICU: an updated metaanalysis. Neonatal Network 2012;31:311-6.

35. Stokes A, Agthe AG, El Metwally D. Music exposure and maturation of late preterm sleep-wake cycles: a randomised crossover trial. Acta Paediatr 2018:107:582-6.

36. van der Heijden MJE, Oliai Araghi S, Jeekel J, et al. Do hospitalized premature infants benefit from music interventions? A systematic review of randomized controlled trials. PLoS One 2016;11:e0161848

37. Shoemark H. Time together: a feasible program to promote parent-infant interaction in the NICU. Music Therapy Perspectives 2018;36:6-16.

38. Bieleninik Łucja, Ghetti C, Gold C. Music therapy for preterm infants and their parents: a meta-analysis. Pediatrics 2016;138:e20160971.

39. Hoffenkamp HN, Tooten A, Hall RAS, et al. The impact of premature childbirth on parental bonding. Evol Psychol 2012;10:147470491201000-561.

40. American Academy of Pediatrics, Age terminology during the perinatal period. Pediatrics 2004;114:1362-4.

41. Haslbeck FB. The interactive potential of creative music therapy with premature infants and their parents: a qualitative analysis. Nord $J$ Music Ther 2014;23:36-70.

42. Haslbeck FB, Bucher H-U, Bassler D, et al. Creative music therapy to promote brain structure, function, and neurobehavioral outcomes in preterm infants: a randomized controlled pilot trial protocol. Pilot Feasibility Stud 2017;3.

43. Loewy J. NICU music therapy: song of kin as critical lullaby in research and practice. Ann N Y Acad Sci 2015;1337:178-85.

44. Rhythm First Sounds:. Breath, Lullaby trainer compendium. NY: Satchnote Armstrong Press, 2016.

45. Abromeit $\mathrm{DH}$, Hanson Abromeit $\mathrm{D}$. The newborn individualized developmental care and assessment program (NIDCAP) as a model for clinical music therapy interventions with premature infants. Music Therapy Perspect 2003;21:60-8.

46. Ettenberger M, Rojas Cárdenas C, Parker M, et al. Family-centred music therapy with preterm infants and their parents in the neonatal intensive care unit (NICU) in Colombia - a mixed-methods study. Nord J Music Ther 2017;26:207-34.

47. Stormark KM, Braarud HC. Infants' sensitivity to social contingency: a "double video" study of face-to-face communication between 2- and 4-month-olds and their mothers. Infant Behavior and Development 2004;27:195-203.

48. Malloch S,Trevarthen C, eds. Communicative musicality: exploring the basis of human companionship. Oxford: Oxford University Press, 2009

49. Beeghly M, Tronick E. Early resilience in the context of Parent-Infant relationships: a social developmental perspective. Curr Probl Pediatr Adolesc Health Care 2011:41:197-201.

50. Stewart K. PATTERNS-A Model for Evaluating Trauma in NICU Music Therapy: Part 1-Theory and Design. Music Med 2009;1:29-40.

51. American Academy of Pediatrics: Committee on Environmental Health. Noise: a hazard for the fetus and newborn. Pediatrics 1997;100:724-7.

52. White RD, Smith JA, Shepley MM, et al. Recommended standards for newborn ICU design, eighth edition. J Perinatol 2013;33 Suppl 1:S2-S16.

53. Haslbeck FB. Music therapy with preterm infants - theoretical approach and first practical experience. Music Therapy Today 2004;5:1-15.

54. Shaker CS. Cue-based co-regulated feeding in the neonatal intensive care unit: supporting parents in learning to feed their preterm infant. Newborn and Infant Nursing Reviews 2013;13:51-5.

55. Markestad T, Halvorsen B. Faglige retningslinjer for oppfølging av for tidlig fødte barn. Oslo: Sosial- og helsedirektoratet, 2007.

56. World Health Organization. Process of translation and adaptation of instruments, 2018. Available: http://www.who.int/substance_abuse/ research_tools/translation/en/\#

57. Lotzin A, Lu X, Kriston L, et al. Observational tools for measuring Parent-Infant interaction: a systematic review. Clin Child Fam Psychol Rev 2015;18:99-132.

58. Perrelli JGA, Zambaldi CF, Cantilino A, et al. [Mother-child bonding assessment tools]. Rev Paul Pediatr 2014;32:257-65.

59. Brockington IF, Oates J, George S, et al. A screening questionnaire for mother-infant bonding disorders. Arch Womens Ment Health 2001;3:133-40.

60. Bayley N. Bayley Scales of Infant and Toddler Development. 3 edn. San Antonio: Pearson, 2006. 
61. Luttikhuizen dos Santos ES, de Kieviet JF, Königs M, et al. Predictive value of the Bayley scales of infant development on development of very preterm/very low birth weight children: a meta-analysis. Early Hum Dev 2013;89:487-96.

62. Squires J, Bricker D. Ages \& Stages Questionnaires. Third edition (ASQ-3). Baltimore: Brookes Publishing Company, 2009.

63. Squires J, Bricker D, Potter L. Revision of a parent-completed development screening tool: ages and stages questionnaires. $J$ Pediatr Psychol 1997;22:313-28.

64. Squires J, Bricker D, Twombly E, et al. Ages \& Stages Questionnaires: Social-Emotional. Second edition(ASQ:SE-2). Baltimore: Brookes Publishing Company, 2015.

65. Squires J, Bricker D, Heo K, et al. Identification of social-emotional problems in young children using a parent-completed screening measure. Early Child Res Q 2001:16:405-19.

66. McBride HLet al. The Edinburgh Postnatal Depression Scale (EPDS): a review of the reported validity evidence. In: Zumbo BD, Chan EK, eds. Validity and validation in social, behavioral, and health sciences. Switzerland: Springer International Publishing, 2014: 157-74.

67. Spitzer RL, Kroenke K, Williams JBW, et al. A brief measure for assessing generalized anxiety disorder. Arch Intern Med 2006;166:1092-7.
68. Berry JO, Jones WH. The parental stress scale: initial psychometric evidence. J Soc Pers Relat 1995;12:463-72.

69. Tsivos Z-L, Calam R, Sanders MR, et al. A pilot randomised controlled trial to evaluate the feasibility and acceptability of the baby triple $\mathrm{P}$ positive parenting programme in mothers with postnatal depression. Clin Child Psychol Psychiatry 2015;20:532-54.

70. O'Mahen HA, Richards DA, Woodford J, et al. Netmums: a phase II randomized controlled trial of a guided Internet behavioural activation treatment for postpartum depression. Psychol Med 2014;44:1675-89.

71. Duerr Aet al. Årstabeller for Medisinsk fødselsregister 2012. In: Fødsler in Norge. Utgitt av Folkehelseinstituttet, 2014.

72. van Buuren S. Flexible imputation of missing data. Interdisciplinary Statistics Series. In: Boca Raton. FL: CRC Press / Taylor \& Francis Group, 2012.

73. Filmer D, Pritchett LH. Estimating wealth effects without expenditure data--or tears: an application to educational enrollments in states of India. Demography 2001;38:115-32.

74. Thorpe KE, Zwarenstein M, Oxman AD, et al. A pragmaticexplanatory continuum indicator summary (Precis): a tool to help trial designers. J Clin Epidemiol 2009;62:464-75. 